\title{
Factors affecting surgical margin positivity in robotic assisted radical prostatectomy
}

\author{
Mustafa Yuksel, Kaan Karamık, Hakan Anıl, Ekrem Islamoglu, Mutlu Ates, Murat Savas \\ Antalya Training and Research Hospital, Antalya, Turkey.
}

\begin{abstract}
Summary Objectives: After radical prostatectomy, surgical margin positivity is an important indicator of biochemical recurrence and progression. In our study we want to compare the surgical margin positivity rates for retropubic radical prostatectomy $(R R P)$ and robotic assisted radical prostatectomy (RALP) and investigate the factors affecting surgical margin positivity in RALP. Materials and methods: Data from 78 RRP and 62 RALP patients operated from 2011 May to 2016 March were retrospectively screened. Patients in both groups were compared in terms of age, postop hematocrit reduction, hospital stay, duration of follow-up, surgical margin positivity, biochemical recurrence and oncologic parameters. In RALP group it was searched the relationship between the surgical margin positivity and prostate specific antigen (PSA), positive biopsy core, biopsy Gleason scoring, pathologic stage and Gleason scoring, lymph node positivity, lymphovascular and perineural invasion, extracapsular extension, seminal vesicle invasion, prostate weight.

Results: Patients in the RALP group had lower postop hematocrit reduction and shorter hospital stay $(p<0.001)$. There was no difference in surgical margin positivity between RALP and RRP groups (37.1\% vs. $29.5 \%, p=0.341$ ). In RALP group there was a correlation between surgical margin positivity and positive biopsy core number $(p=0.011)$, pathologic stage $(p<0.001)$ and Gleason score $(p<0.001)$, EAU risk classification ( $p=0.001)$, seminal vesicle invasion $(p=0.045)$, extraprostatic extension $(p<0.001)$. There was no correlation between prostate weight $(p=0.896)$, PSA ( $p=0.220)$, biopsy Gleason score $(p=0.266)$, lymph node positivity $(p=0.140)$, perineural $(p=0.103)$ and lymphovascular invasion ( $p=0.92)$ with surgical margin positivity. Conclusions: Positive biopsy core number, pathological stage and Gleason score, EAU risk classification, seminal vesicle invasion and extraprostatic extension are correlated with surgical margin positivity in RALP.
\end{abstract}

KEY WORDS: Prostatectomy; Surgical margin; Prostate cancer.

Submitted 7 November 2016; Accepted 11 January 2017

\section{INTRODUCTION}

Prostate cancer is the $2^{\text {nd }}$ most common cancer type and is the $5^{\text {th }}$ cause of cancer-related death in men (1).

The gold standard treatment for localized prostate cancer currently is radical prostatectomy (2). Much knowledge and experience of the open technique has been gained since radical prostatectomy was modernized by Walsh (3) in 1982. The robotic technique for radical prostatectomy was first used by Binder and Kramer (4) in 2001 and is being applied at greater rates through time. Among the advantages of robotic assisted radical prostatectomy (RARP) are technical details such as appropriate mimicking of the wrist maneuvers used in open surgery, 3-dimensional imaging and ability to perform surgery under magnification. In 2008, the open technique was chosen for $44 \%$ and the robotic technique was chosen for $53 \%$ of the procedures of radical prostatectomy in the USA (5). When the literature is investigated, it appears the hospital stay and transfusion rates are lower for the robotic technique compared to the open technique, though when functional and oncologic results are investigated contradictory results are found (6-9).

Additionally the high cost associated with robotic radical prostatectomy has led to questions about the necessity for use of this technique in developing countries such as ours (10). Surgical margin positivity after radical prostatectomy is one of the important causes of biochemical recurrence and progression. When comparing retropubic radical prostatectomy (RRP) and RARP one of the important topics of interest is the effect on surgical margin positivity.

In this study we compared the surgical margin positivity rates of RRP and RARP and aimed to investigate the factors affecting surgical margin positivity in RARP.

\section{Materials AND Methods}

The data belonging to 173 patients who underwent RRP or RARP for prostate cancer, without neo-adjuvant therapy, at our center from May 2011 to March 2016 were retrospectively scanned. Our study was in accordance with the Helsinki Declaration and did not gain ethics committee permission as it included retrospective data. While RRP was performed with the classic retropubic technique, the robotic technique used the da Vinci robotic surgical system (Intuitive Surgical, Sunnyvale, CA, USA) with 5 port transperitoneal approach. The operations were performed by 3 different surgeons experienced in open surgery and inexperienced in robotic surgery. Patients with lymph node metastasis risk above 5\% according to the Briganti nomogram (11) had extended lymph node dissection (2) performed. For low risk prostate cancer (Tlc, PSA $<10$, Gleason $<7$ ) patients, a nerve-sparing approach was chosen. Patients who underwent RRP had a urethral Foley catheter inserted for 2 weeks, while RARP patients had a catheter inserted for 1 week. The patient age, prostate 
specific antigen (PSA), positive core number on biopsy, biopsy Gleason scoring, hospital stay, surgical pathology stage according to TNM classification, surgical Gleason scoring, lymph node positivity, surgical margin condition, lymphovascular and perineural invasion, extracapsular extension and seminal vesicle invasion conditions, prostate weight, and biochemical recurrence during follow-up were recorded. Patients were classified as low risk, moderate risk and high risk based on biopsy pathology and PSA in accordance with the European Association of Urology (EAU) prostate cancer guidelines.

According to TNM surgical staging, patients were divided into T2 and T3-T4. Biochemical recurrence was defined as postoperative PSA rising above $0.2 \mathrm{ng} / \mathrm{ml}$. Thirty-three patients with incomplete data were excluded from the study. Early oncologic results of 78 RRP and 62 RARP patients were compared in relation to the recorded parameters. Additionally, the factors affecting surgical margin positivity in RARP patients were researched.

\section{Statistical analysis}

Descriptive statistics of data are given as mean, standard deviation, median, frequency and percentage. The distribution of variables was measured with the Kolmogorov Smirnov test. Quantitative data were analyzed with the Mann-Whitney U test. Analysis of qualitative data used the chi-square test, with the Fisher test used when chi-square test conditions were not valid. Analyses were completed using the SPSS 22.0 program (SPSS, Chicago, IL, USA).

A value of $\mathrm{p}<0.05$ was accepted as statistically significant.

\section{Results}

The data of patients undergoing retropubic radical prostatectomy and robotic assisted radical prostatectomy are shown in Table 1. The follow-up duration for RRP group patients was clearly longer than for RARP patients $(\mathrm{p}<0.05)$. Patients in the RARP group had less postop hematocrit reduction and shorter hospital stays ( $\mathrm{p}<0.05$ ). While there was no difference between patients of the two groups in terms of biopsy Gleason score and PSA ( $p>0.05$ ), surgical stage and Gleason score in the RRP group were higher $(p<0.05)$. Though surgical margin positivity was observed at a higher rate in the RARP group compared to the RRP group ( $37.1 \%$ vs. $29.5 \%$ ), this difference did not reach statistical significance $(p=0.341)$. Additionally, biochemical recurrence was observed at a higher rate in the RRP group (26.9\% vs. $16.1 \%, \mathrm{p}=0.127)$. The factors affecting surgical margin positivity in patients treated by the robotic assisted radical prostatectomy group are investigated in Table 2. When patients in the RARP group are investigated in terms of surgical margin positivity, a correlation was found between biopsy positive core number, EAU risk classification, surgical stage, surgical Gleason degree, seminal vesicle invasion and extraprostatic extension with surgical margin positivity $(\mathrm{p}<0.05)$. Additionally, surgical margin positivity increased the incidence of biochemical recurrence $(\mathrm{p}<0.05)$.

\section{Discussion}

Surgical margin positivity in radical prostatectomy is one of the important factors affecting disease recurrence and progression. D'Amico et al. reported that the 2 year biochemical recurrence rate in patients with surgical margin positivity (45-55\%) was higher compared to patients with organ-limited disease (15-25\%) (12). In our study investigating the data of 140 radical prostatectomy patients, surgical margin positivity in RARP group patients increased biochemical recurrence. Though surgical stage and surgical Gleason degree were higher in RRP patients, surgical margin positivity in RARP patients was still observed at a higher rate (although difference did not reach statistical significance). We believe this situation may be linked to still being in the learning stage for robotic radical prostatectomy operations.

Biochemical recurrence rates were observed to be higher in the RRP group (although difference was not statistically significant), but we believe that this observation may be due to the longer follow up period in the RRP group.

When factors affecting surgical margin positivity in robotic radical prostatectomy are investigated, the results of literature appears to be very complex. A study by Liss et al. in RARP patients, found a correlation of surgical margin positivity with PSA $(\mathrm{P}=0.012)$ and PSA density $(\mathrm{P}=0.005)$ 
Table 2.

Factors affecting surgical margin positivity in robotic assisted radical prostatectomy.

\begin{tabular}{|c|c|c|c|c|c|c|c|c|c|}
\hline & \multirow{4}{*}{\multicolumn{3}{|c|}{$\begin{array}{c}\begin{array}{c}\text { Surgical margin (-) } \\
\text { robotik prostatectomy }\end{array} \\
\mathrm{n}=39 \\
\text { Mean } \pm \text { S.D } / \mathrm{n}-\% \text { Med }\end{array}$}} & \multirow{4}{*}{\multicolumn{3}{|c|}{$\begin{array}{c}\begin{array}{c}\text { Surgical margin (+) } \\
\text { Robotic prostatectomy }\end{array} \\
n=23 \\
\text { Mean } \pm \text { S.D } / \mathrm{n}-\% \text { Med }\end{array}$}} & \multirow{4}{*}{\multicolumn{2}{|c|}{ p }} \\
\hline & & & & & & & & & \\
\hline & & & & & & & & & \\
\hline & & & & & & & & & \\
\hline \multicolumn{2}{|l|}{ Age (year) } & 63.2 & \pm 6.6 & 65.0 & 63.9 & \pm 7.3 & 66.0 & 0.672 & $\bar{m}$ \\
\hline \multicolumn{2}{|c|}{ Positive biopsy core number } & 3.3 & \pm 2.2 & 3.0 & 5.3 & \pm 3.0 & 5.0 & 0.011 & $\bar{m}$ \\
\hline \multirow[t]{4}{*}{$\mathrm{PSA}(\mathrm{ng} / \mathrm{ml})$} & $0-4$ & 3 & $7.7 \%$ & & 1 & $4.3 \%$ & & & \\
\hline & $4-10$ & 25 & $64.1 \%$ & & 12 & $52.2 \%$ & & & $x^{2}$ \\
\hline & $10-20$ & 11 & $28.2 \%$ & & 5 & $21.7 \%$ & & 0.220 & $x^{2}$ \\
\hline & $20^{>}$ & 0 & $0.0 \%$ & & 5 & $21.7 \%$ & & & \\
\hline \multirow[t]{3}{*}{ Biopsy gleason } & $\leq 6$ & 29 & $74.4 \%$ & & 14 & $60.9 \%$ & & & \\
\hline & 7 & 10 & $25.6 \%$ & & 6 & $26.1 \%$ & & 0.266 & $x^{2}$ \\
\hline & $8-10$ & 0 & $0.0 \%$ & & 3 & $13.0 \%$ & & & \\
\hline \multirow{3}{*}{\multicolumn{2}{|c|}{$\begin{array}{ll}\text { Risk } & \text { High risk } \\
\text { Intermediate risk } \\
\text { Low risk }\end{array}$}} & 0 & $0.0 \%$ & & 7 & $30.4 \%$ & & & \\
\hline & & 19 & $48.7 \%$ & & 9 & $39.1 \%$ & & 0.001 & $x^{2}$ \\
\hline & & 20 & $51.3 \%$ & & 7 & $30.4 \%$ & & & \\
\hline \multirow[t]{3}{*}{ Prostatectomy gleason } & $\leq 6$ & 21 & $53.8 \%$ & & 2 & $8.7 \%$ & & & \\
\hline & 7 & 14 & $35.9 \%$ & & 20 & $87.0 \%$ & & 0.000 & $x^{2}$ \\
\hline & $8-10$ & 4 & $10.3 \%$ & & 1 & $4.3 \%$ & & & \\
\hline \multirow[t]{2}{*}{ Lymphovascular invasion } & $(-)$ & 30 & $76.9 \%$ & & 13 & $56.5 \%$ & & & $x^{2}$ \\
\hline & $(+)$ & 9 & $23.1 \%$ & & 10 & $43.5 \%$ & & 0.092 & $A^{n}$ \\
\hline \multirow[t]{2}{*}{ Perineural invasion } & $(-)$ & 10 & $25.6 \%$ & & 2 & $8.7 \%$ & & 0103 & $x^{2}$ \\
\hline & $(+)$ & 29 & $74.4 \%$ & & 21 & $91.3 \%$ & & 0.103 & $\hat{n}$ \\
\hline \multirow[t]{2}{*}{ Lymph node invasion } & $(-)$ & 38 & $97.4 \%$ & & 20 & $87.0 \%$ & & 0140 & $x^{2}$ \\
\hline & $(+)$ & 1 & $2.6 \%$ & & 3 & $13.0 \%$ & & 0.140 & \\
\hline \multirow[t]{2}{*}{ Seminal vesicle invasion } & $(-)$ & 35 & $89.7 \%$ & & 16 & $69.6 \%$ & & 0.045 & $x^{2}$ \\
\hline & $(+)$ & 4 & $10.3 \%$ & & 7 & $30.4 \%$ & & 0.045 & $\hat{n}$ \\
\hline \multirow[t]{2}{*}{ Biochemical recurrence } & $(-)$ & 37 & $94.9 \%$ & & 15 & $65.2 \%$ & & & $x^{2}$ \\
\hline & $(+)$ & 2 & $5.1 \%$ & & 8 & $34.8 \%$ & & 0.002 & \\
\hline \multirow[t]{7}{*}{ Stage } & T2 & 29 & $74.4 \%$ & & 4 & $17.4 \%$ & & ב000 & $x^{2}$ \\
\hline & T3 & 10 & $25.6 \%$ & & 19 & $82.6 \%$ & & 0.000 & $x^{*}$ \\
\hline & T2A & 10 & $25.6 \%$ & & 0 & $0.0 \%$ & & & \\
\hline & T2B & 1 & $2.6 \%$ & & 0 & $0.0 \%$ & & & \\
\hline & $\mathrm{T} 2 \mathrm{C}$ & 18 & $46.2 \%$ & & 4 & $17.4 \%$ & & & \\
\hline & T3A & 6 & $15.4 \%$ & & 12 & $52.2 \%$ & & & \\
\hline & T3B & 4 & $10.3 \%$ & & 7 & $30.4 \%$ & & & \\
\hline \multirow[t]{2}{*}{ Extraprostatic extension } & $(-)$ & 29 & $74.4 \%$ & & 0 & $0.0 \%$ & & 0.000 & $x^{2}$ \\
\hline & $(+)$ & 10 & $25.6 \%$ & & 23 & $100 \%$ & & 0.000 & \\
\hline
\end{tabular}

ed 282 RARP and 621 RRP operations and found no difference between the 2 techniques in terms of surgical margin positivity (24.5\% vs. 23.1\%, p = 0.51).

A meta-analysis by Soorikumaran et al. of data considering 22.393 patients showed that surgical margin positivity was higher in the open radical prostatectomy group $(22.8 \%)$ compared to the RARP group (13.8\%) (18). When assessing the results of this study, it should be noted that the patients in the open radical prostatectomy group had higher rates of high risk prostate cancer. Tewari et al. investigated data from 286.876 radical prostatectomy patients obtained from 400 studies and found no difference between robotic RP and open RP in terms of surgical margin positivity (19). Additionally, surgical margin positivity appeared to be lower in robotic RP compared to laparoscopic RP.

Experience is one of the most important factors affecting oncologic and functional results in robotic-aided radical prostatectomy. A study conducted in 2009 on the effects of surgical experience on RARP (20) investigated the results of 700 RARP operations. This study compared the results of the operations from 0 300, 300-500 and 500-700 and

but no correlation was found with clinical stage and biopsy Gleason score (13). Ficarra et al. found a correlation between surgical margin positivity and biopsy Gleason score, pathologic stage and Gleason score and extraprostatic extension but did not find a correlation with PSA (14). Coelho et al. found a correlation between pathologic stage and pathologic Gleason score with surgical margin positivity, while no correlation was found with PSA, biopsy Gleason score and biopsy positive core number (15). In our study we found a correlation of positive biopsy core number, pathologic stage and Gleason score, EAU risk classification, seminal vesicle invasion and extraprostatic extension with surgical margin positivity in RARP. Additionally, no correlation was found with PSA, biopsy Gleason score, perineural and lymphovascular invasion and lymph node positivity. In the literature, there are some studies stating that a small prostate neck may increase the risk of surgical margin positivity (16), though in our study there was no correlation found between prostate weight and surgical margin positivity in RARP patients. When studies of the literature comparing surgical margin positivity rates in robotic assisted radical prostatectomy and retropubic radical prostatectomy are considered, contradictory results are found. A study in 2015 (17) investigat- observed that as experience increased the surgical margin positivity rates decreased. A study by Kwon et al. investigating 286 patients compared the results of 165 RARP performed by surgeons with no experience of RARP and 121 RARP operations performed by surgeons with experience of RARP (21). In this study, experience of RARP appeared to directly affect surgical margin positivity (24\% vs. $34.6 \%, P=0.05)$. As we are still in the early stages of RARP surgery, we believe that our surgical margin positivity rates would decrease in course of time.

There are studies investigating the effects of surgical technique on surgical margin positivity in robotic assisted radical prostatectomy. A study in 2009 did not find an effect on surgical margin positivity for nerve-sparing extrafascial and intrafascial techniques (22). Chung et al. (23) investigated the effect of transperitoneal and extraperitoneal robotic prostatectomy on surgical margin positivity and obtained similar rates for both techniques. Similarly bladder neck-sparing did not have an effect on surgical margin positivity (24). In a study published in 2009 (25) incision after suture ligation of the dorsal venous complex had greater rates of apical surgical margin positivity compared to only cold incision $(\mathrm{p}=0.02)$. One of the points where RARP appears to be superior to 
RRP is the low rate of postoperative blood transfusion and shorter hospital stay $(26,27)$. In our study, the RARP group patients had lower postoperative hemoglobin decrease and shorter hospital stays compared to patients in the RRP group.

One of the limitations of our study is that the functional results of both radical prostatectomy techniques were not included as it was retrospective. Additionally due to the retrospective nature of the study, we could not investigate the effects on surgical margin positivity of standardizing the surgical technique. In addition to these points, our results reflect results from the learning stage. We believe that a future update will negate this problem.

\section{Conclusions}

Biopsy positive core number, surgical stage and Gleason degree, EAU risk classification, seminal vesicle invasion and extraprostatic extension are correlated with surgical margin positivity in RARP.

\section{REFERENCES}

1. World Cancer Report 2014. World Health Organization. 2014. Chapter 1.1.

2. Mottet N, Bellmunt J, Bolla M, et al. EAU-ESTRO-SIOG Guidelines on Prostate Cancer. Part 1: Screening, Diagnosis, and Local Treatment with Curative Intent. Eur Urol. 2016; pii: S03022838(16)30470-5.

3. Walsh PC, Donker PJ. Impotence following radical prostatectomy: insight into etiology and prevention. J Urol. 1982; 128:492-7.

4. Binder J, Kramer W. Robotically assisted laparoscopic radical prostatectomy. BJU Int. 2001; 87:408-10.

5. Yu HY, Hevelone ND, Lipsitz SR, et al. Use, costs and comparative effectiveness of robotic assisted, laparoscopic and open urological surgery. J Urol. 2012; 187:1392-8.

6. Rocco B, Matei DV, Melegari S, et al. Robotic vs open prostatectomy in a laparoscopically naive centre: a matched-pair analysis. BJU Int. 2009; 104:991-5.

7. Robertson C, Close A, Fraser C, et al. Relative effectiveness of robot-assisted and standard laparoscopic prostatectomy as alternatives to open radical prostatectomy for treatment of localised prostate cancer: a systematic review and mixed treatment comparison meta-analysis. BJU Int. 2013; 112:798-812.

8. Farnham SB, Webster TM, Herrell SD, Smith JA Jr. Intraoperative blood loss and transfusion requirements for roboticassisted radical prostatectomy versus radical retropubic prostatectomy. Urology. 2006; 67:360-3

9. Thompson JE, Egger S, Böhm M, et al. Superior quality of life and improved surgical margins are achievable with robotic radical prostatectomy after a long learning curve: a prospective single-surgeon study of 1552 consecutive cases. Eur Urol. 2014; 65:521-31.

10. Close A, Robertson C, Rushton S, et al. Comparative cost-effectiveness of robot-assisted and standard laparoscopic prostatectomy as alternatives to open radical prostatectomy for treatment of men with localised prostate cancer: a health technology assessment from the perspective of the UK National Health Service. Eur Urol. 2013; 64:361-9.

11. Briganti A, Larcher A, Abdollah F, et al. Updated nomogram predicting lymph node invasion in patients with prostate cancer undergoing extended pelvic lymph node dissection: the essential importance of percentage of positive cores. Eur Urol. 2012; 61:480-7.

12. D'Amico AV, Whittington R, Malkowicz SB, et al. A multivariate analysis of clinical and pathological factors that predict for prostate specific antigen failure after radical prostatectomy for prostate cancer. J Urol. 1995; 154:131-8.

13. Liss M, Osann K, Ornstein D. Positive surgical margins during robotic radical prostatectomy: a contemporary analysis of risk factors. BJU Int. 2008; 102:603-7.

14. Ficarra V, Novara G, Secco S, et al. Predictors of positive surgical margins after laparoscopic robot assisted radical prostatectomy. J Urol. 2009; 182:2682-8.

15. Coelho RF, Chauhan S, Orvieto MA, et al. Predictive factors for positive surgical margins and their locations after robot-assisted laparoscopic radical prostatectomy. Eur Urol. 2010; 57:1022-9.

16. Link BA, Nelson R, Josephson DY, et al. The impact of prostate gland weight in robot assisted laparoscopic radical prostatectomy. J Urol. 2008; 180:928-32.

17. Alemozaffar M, Sanda M, Yecies D, et al. Benchmarks for operative outcomes of robotic and open radical prostatectomy: results from the HealthProfessionals Follow-up Study. Eur Urol. 2015; 67:432-8.

18. Sooriakumaran P, Srivastava A, Shariat SF, et al. A multinational, multi-institutional study comparing positive surgical margin rates among 22393 open, laparoscopic, and robot-assisted radical prostatectomy patients. Eur Urol. 2014; 66:450-6.

19. Tewari A, Sooriakumaran P, Bloch DA, et al. Positive surgical margin and perioperative complication rates of primary surgical treatments for prostate cancer: a systematic review and meta-analysis comparing retropubic, laparoscopic, and robotic prostatectomy. Eur Urol. 2012; 62:1-15.

20. Zorn KC, Wille MA, Thong AE, et al. Continued improvement of perioperative, pathological and continence outcomes during 700 robotassisted radical prostatectomies. Can J Urol. 2009; 16:4742-9.

21. Kwon EO, Bautista TC, Jung $H$, et al. Impact of robotic training on surgical and pathologic outcomes during robot-assisted laparoscopic radical prostatectomy. Urology. 2010; 76:363-8.

22. Shikanov S, Woo J, Al-Ahmadie H, et al. Extrafascial versus interfascial nerve-sparing technique for robotic-assisted laparoscopic prostatectomy: comparison of functional outcomes and positive surgical margins characteristics. Urology. 2009; 74:611-6.

23. Chung JS, Kim WT, Ham WS, et al. Comparison of oncological results, functional outcomes, and complications for transperitoneal versus extraperitoneal robotassisted radical prostatectomy: a single surgeon's experience. J Endourol. 2011; 25:787-92.

24. Freire MP, Weinberg AC, Lei Y, et al. Anatomic bladder neck preservation during robotic-assisted laparoscopic radical prostatectomy: description of technique and outcomes. Eur Urol. 2009; 56:972-80

25. Guru KA, Perlmutter AE, Sheldon MJ, et al. Apical margins after robot-assisted radical prostatectomy: does technique matter? J Endourol. 2009; 23:123-7.

26. Farnham SB, Webster TM, Herrell SD, Smith JA Jr. Intraoperative blood loss and transfusion requirements for roboticassisted radical prostatectomy versus radical retropubic prostatectomy. Urology. 2006; 67:360-3.

27. Nelson B, Kaufman M, Broughton G, et al. Comparison of length of hospital stay between radical retropubic prostatectomy and robotic assisted laparoscopic prostatectomy. J Urol. 2007; 177:929-31.

\section{Correspondence}

Mustafa Yuksel, MD (Corresponding Author) - drmustafayuksel@outlook.com Kaan Karamik, MD - Hakan Anil, MD - Ekrem Islamoglu, MD

Mutlu Ates, MD - Murat Savas, MD

Department of Urology, Antalya Training and Research Antalya EğitimAraştırmaHastanesi, VarlıkMh.

07050 Muratpaşa/Antalya, Turkey 